\title{
Ecotoxicity assessment of camphor oxime using Microtox assay - preliminary research
}

\author{
Ewa Kielka ${ }^{1}$, Agata Siedlecka ${ }^{2, *}$, Mirela Wolf ${ }^{2}$, Sylwia Stróżak $^{2}$, Katarzyna Piekarska ${ }^{2}$, \\ Daniel Strub ${ }^{1}$ \\ ${ }^{1}$ Wrocław University of Science and Technology, Faculty of Chemistry, Wyb. Wyspiańskiego 27, \\ 50-370, Wrocław \\ ${ }^{2}$ Wrocław University of Science and Technology, Faculty of Environmental Engineering, \\ Wyb. Wyspiańskiego 27, 50-370, Wrocław
}

\begin{abstract}
The ecotoxicity assessment of new substances is crucial for their considered launch on the market. Due to various properties of chemical compounds, however, the application of biotests can be challenging. In the case of insoluble compounds, a proper, non-toxic solvent must be applied. The presented preliminary results demonstrate the extremely high ecotoxicity of camphor and camphor oxime, assessed by means of the Microtox system. A $81.9 \%$ Basic Test with 14 dilutions was applied. The ecotoxicity of a solvent (i.e. DMSO) was also assessed. The initial concentration of substances was $5 \% . \mathrm{EC}_{50}$ values reached $0.00023 \%$ and $0.00007 \%$ for camphor and camphor oxime, respectively, after $15 \mathrm{~min}$. of incubation of the samples with bioluminescent bacteria. The ecotoxicity of the solvent remained negligible. Due to the extremely high ecotoxicity values of the tested substances, another range of concentrations needs to be applied in future tests to confirm the results.
\end{abstract}

\section{Introduction}

After chemical synthesis, the possibility of launch of a new product on the market is often considered. To accomplish the procedure, many tests regarding its quality, purity, physicalchemical properties, etc. are required. Moreover, the toxicity and ecotoxicity of each new compound need to be tested to evaluate potential environmental risk, as determined in the European Union Regulation concerning the Registration, Evaluation, Authorisation, and Restriction of Chemicals (REACH) [1]. The entire production process may indeed have some negative effects on people and the environment. Therefore, the LCA (life-cycleassessment) should not be neglected either [2].

A new compound, namely camphor oxime, was synthesised at the Department of Bioorganic Chemistry, Faculty of Chemistry, Wrocław University of Science and Technology, from camphor. Camphor oxime can become a substrate for the synthesis of fragrances which can be further used in perfumes, cosmetics, and household chemicals. The search for novel fragrances is needed, because many of the currently used ones may be withdrawn from the market. A good example is musk which revealed to possess some toxic

* Corresponding author: agata.siedlecka@pwr.edu.pl 
properties [3]. The novel fragrances should exhibit durability in strongly alkaline environment, determined by their future application. The compounds or their derivatives may be supplied to the environment, so their ecotoxicology must be known, as it was proven in the case of citral oxime ethers [4]. Although up to several dozen new compounds are assumed to be synthetised, camphor oxime is a good model substance in preliminary ecotoxicology studies due to its chemical-physical properties. The ecotoxicity of each novel substance, however, will be tested.

The chemical structures of camphor oxime (intermediate) and camphor (substrate) are presented in Figure 1.

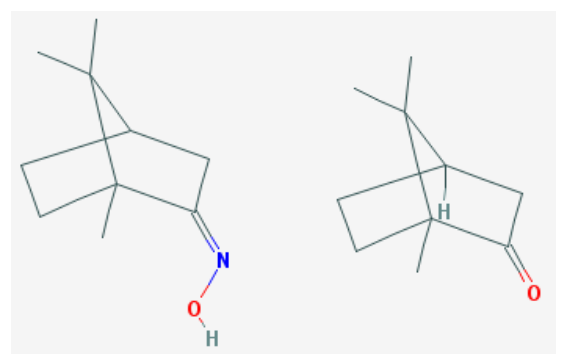

Fig. 1. Camphor oxime (left) and camphor (right) chemical structures [5].

Because each alteration in the chemical structure of a substance may lead to changes in its properties, the toxicity of camphor oxime may differ from the toxicity of camphor. Oral ingestion of low doses of camphor is evidenced to potentially cause significant toxicity. Ingestion of $700 \mathrm{mg}-1 \mathrm{~g}$ of camphor can cause death [6]. The substance is known to affect some sex hormones in rats, but its inhibitory effect on sexual activity is not confirmed [7]. To the authors' best knowledge, however, little is known about the ecotoxicity of camphor. Because camphor oxime is a new compound, data on its ecotoxicity are unavailable.

In order to evaluate whether the chemical transformation of camphor to camphor oxime makes the product more or less toxic, the ecotoxicity assessment of both products should be conducted and compared.

The determination of the acute ecotoxicity of a substance in a fast and reliable wayfrequently involves the application of the Microtox assay [8]. The principle of the system is based on the inhibition of luminescence in marine bacteria Allivibrio fischeri as a result of toxic action of chemicals $[9,10]$. It is a fast, sensitive, and cost-effective method that can indicate the level of ecotoxicity of the tested substance. Although a complete ecotoxicology assessment requires biotests on organisms at each trophic level $[1,11]$, Microtox is often chosen as the first biotest in a biotest battery based on speed and cost consideration [12].

The main obstacle in ecotoxicology assessment of organic substances with Microtox system is their solubility. Because Micrtotox was developed primarily for liquids (water, wastewaters), it requires a solution of the tested substance [13]. The solid-phase protocol of the Microtox system is dedicated for hydrophilic substances, whereas camphor and camphor oxime are insoluble in water. This obstacle was overcome by other researchers by dissolving hydrophobic substances in acetone [9] or/and DMSO (dimethyl sulfoxide) $[14,15]$. DMSO is used in cytotoxicity assays as well [16]. Because the solvents possess some toxicity themselves, the analysis is based on the comparison of the ecotoxicity effect of the solvent, and solvent with dissolved substance [17].

This study presents preliminary research on the ecotoxicity of a new substance, namely camphor oxime, as well as camphor, to determine the level of ecotoxicity of the converted substrate and its product. The biotest of choice was the Microtox system, providing fast results. 


\section{Materials and methods}

\subsection{Preparation of samples}

Samples of camphor oxime and camphor were provided by the Department of Bioorganic Chemistry, Faculty of Chemistry, Wrocław University of Science and Technology. Because each of the substances was insoluble in water, their solubility in acetone and DMSO was determined. Both substances were determined to be easily dissolved in acetone and DMSO. Therefore, the $\mathrm{EC}_{50}$ value of the solvents was measured in Microtox $81.9 \%$ Basic Test. Because DMSO proved to be less toxic than acetone for marine bacteria Alivibrio fischeri in Microtox $81.9 \%$ Basic Test [data not published], it was the solvent of choice. The same observation on the ecotoxicity of acetone and DMSO was made by Bogaerts et al. [14]. Because the $\mathrm{EC}_{50}$ value of camphor oxime was completely unknown, $5 \%$ solutions in DMSO were prepared and tested.

\subsection{Microtox toxicity analysis}

The Microtox toxicity analysis is based on bioluminescence of marine bacteria Aliibrio fischeri (former Vibrio fischeri [18]). Luminescent bacteria emit light when they are in optimal environmental conditions. The presence of toxic substance causes a change in the cellular state, rapidly reflected in a decrease of light emission [17]. The Microtox $81.9 \%$ Basic Test with 14 double dilutions was chosen to provide a wider range of tested concentrations due to the fact that the ecotoxicity of camphor oxime was completely unknown. The ecotoxicity of samples dissolved in DMSO and pure DMSO (as a background) were measured. Furthermore, the protocol with saccharose was applied.

\subsection{Calculations}

Luminescence intensity was measured by means of a Microtox ${ }^{\mathrm{TM}}$ Model 500 Analyser at 0 -, 5-, and 15-min. exposure time. For the calculations of percent inhibition of light emission, the following equations were used $[13,17]$ :

$$
\begin{gathered}
R_{t}=\frac{I_{c t}}{I_{c 0}} \\
\Gamma_{t}=\frac{R_{t} \times I_{s 0}}{I_{s t}}-1 \\
\% \text { effect }=\frac{\Gamma_{t}}{1+\Gamma_{t}} \times 100
\end{gathered}
$$

where $R_{t}$ is a correction for any inhibition induced by a negative control sample (blank cuvette), and $I_{c 0}$ and $I_{c t}$ are absolute light intensities produced by negative control at time $t$ and at initial time 0 , respectively. $I_{s 0}$ and $I_{s t}$ are light intensities produced by a sample at time $\mathrm{t}$ and at initial time 0 , respectively. $\Gamma_{t}$ is the ratio of light lost at time $t$ to light remaining at time $t$, which can be convert to \% effect. The final expression of the toxic response of the samples is presented as $\mathrm{EC}_{50}$ which is the Effective Concentration of the samples reducing light emission by $50 \%$, and estimated from the linear regression of the log of each concentration level of the contaminant versus percent inhibition ( $\%$ effect), as described in [17]. 
The concentrations of the sample used in the calculations took into account the initial dissolving of the sample in DMSO. Therefore, the initial concentration was

$$
5 \times 81.9 / 100=4.095 \%
$$

\section{Results and discussion}

Despite the easiness of dissolving both tested substances in acetone and DMSO, a precipitate formed during the test. This was probably due to the high concentration of $\mathrm{NaCl}$ in solutions used in the Microtox protocol (2\% in Diluent and 22\% in OAS) [13]. Therefore, the modified protocol with saccharose was applied, but, due to the hydrophilic properties of saccharose, the sugar proved insoluble in the final mixture (consisting of camphor oxime in DMSO).

The results of acute ecotoxicity measurements are presented in Table 1 as $\mathrm{EC}_{50}$ and toxicity equivalents (i.e. $1 / \mathrm{EC}_{50}$ ).

Table 1. EC50 and toxicity equivalents of DMSO, camphor oxime dissolved in DMSO, camphor dissolved in DMSO, and trial with saccharose after 5 and $15 \mathrm{~min}$. of exposure of bioluminescent bacteria.

\begin{tabular}{|l|c|c|c|c|}
\hline \multirow{2}{*}{\multicolumn{1}{|c|}{ sample }} & \multicolumn{2}{c|}{$\mathrm{EC}_{50}$} & \multicolumn{2}{c|}{$1 / \mathrm{EC}_{50}$} \\
\cline { 2 - 5 } & $\mathrm{t}=5 \mathrm{~min}$ & $\mathrm{t}=15 \mathrm{~min}$ & $\mathrm{t}=5 \mathrm{~min}$ & $\mathrm{t}=15 \mathrm{~min}$ \\
\hline DMSO & $3.85500 \%$ & $2.63100 \%$ & 0.259 & 0.380 \\
\hline DMSO + camphor oxime & $0.00011 \%$ & $0.00007 \%$ & 9090.909 & 14285.714 \\
\hline DMSO + camphor & $0.00025 \%$ & $0.00023 \%$ & 4000.000 & 4347.826 \\
\hline DMSO + camphor oxime with saccharose & $0.00006 \%$ & $0.00006 \%$ & 16666.667 & 16666.667 \\
\hline
\end{tabular}

The results of ecotoxicity measurement of camphor oxime and camphor are extremely high. Therefore, the ecotoxicity of pure solvent, i.e. DMSO, can be considered negligible. This suggests that DMSO is a good solvent for further Microtox investigations, as stated by Bogaerts et al. [14]. Although the chemical structure of camphor oxime is very similar to that of camphor, the transformation of a carbonyl group seems to contribute to an increase in the ecotoxicity of the compound. The obtained results, however, require confirmation by means of a Microtox test with another range of concentrations as well as the entire battery of ecotoxicity biotests on organisms at each trophic level $[11,19]$. Meanwhile, the results can be considered only as preliminary. Moreover, other experimental approaches, such as diluting compound-DMSO solution with water to obtain much lower concentrations, can be implemented in future tests $[12,14,20]$. The formation of a precipitate might also affect the obtained results.

The median lethal concentration $\left(\mathrm{LC}_{50}\right)$, i.e. concentration required to kill half the members of a rat population, is established for camphor, and is equal to $0.5 \mathrm{mg} / \mathrm{ml}$ $\left(1 / \mathrm{LC}_{50}=20\right)$ [21]. Marine bacteria Alivibibio fischeri proved much more sensitive to camphor. Effective concentrations inhibiting the light of $50 \%$ of bacterial population $\left(\mathrm{EC}_{50}\right)$ are much lower. Moreover, camphor oxime proved more toxic than camphor. Because camphor oxime is a new substance, its ecotoxicity cannot be compared with other studies. In the case of camphor, to the authors' best knowledge its ecotoxicity is not established. The compound was assessed mainly in terms of toxicity for humans [6].

Due to the above-mentioned problems with precipitation of the sample during the test, however, the results can be considered only as preliminary. The modification of the Microtox protocol and application of other ecotoxicity biotests with organisms at each trophic level are required to fully conclude on the ecotoxicity and potential impact of camphor oxime on the environment. The primary difficulty with the substance is its 
insoluble property, narrowing down the possibilities of direct testing. The majority of ecotoxicology biotests deal with aquatic organisms, such as Daphnia magna, Lemna minor, or microalgae [22]. The appropriate solvent with low toxicity would aid the performance of the biotests. Such requirements can be fulfilled by ionic liquids, found to be less toxic than conventional solvents such as toluene or chloroform [23]. Another approach can involve biotests concerning the ecotoxicology of sediments, as a biotest with Eisenia fetida or other invertebrates [22]. DMSO, however, may prove to be a sufficient solvent for camphor and camphor oxime in the case of obtaining much lower concentrations of compounds in the solvent. A similar approach was applied in ecotoxicity assessments of citral with fish, Daphnia magna, and algae, where DMSO or DMSO: HCO-40 were used for the preparation of stock solutions [24].

Nevertheless, in order to classify a substance as meeting the toxicity criterion, REACH requires the determination of the long-term non-observed effect concentration (NOEC) for marine or freshwater organisms, which must be less than $0.01 \mathrm{mg} / \mathrm{L}$. Other criteria include: carcinogenicity, mutagenicity, toxicity for reproduction or other evidence of chronic toxicity, as identified by the classifications: $\mathrm{T}, \mathrm{R} 48$, or $\mathrm{Xn}$, according to Directive 67/548/EEC [1]. Therefore, acute ecotoxicity can be regarded as the screening procedure for toxicity assessment. It makes further investigations easier, and allows for the proper choice of range of concentrations. Because mutagenicity is one of the criteria mentioned in $\mathrm{REACH}$, the Ames test seems to be a proper tool for further investigations of camphor oxime properties.

\section{Conclusions}

The launch of new substances on the market is inseparably connected with ecotoxicology assessment. Acute ecotoxicity measurements by means of the Microtox system revealed extremely high $\mathrm{EC}_{50}$ values of camphor oxime and camphor, tested on marine bioluminescent bacteria Aliivibrio fischeri. Moreover, camphor oxime seems to be more toxic than camphor, although the results require confirmation. Other ecotoxicity biotests, engaging the organisms at each trophic level, are required to confirm the obtained results. The insoluble properties of camphor oxime make its ecotoxicity assessment challenging.

This work was supported by project "Synthesis of new fragrances from raw materials of a natural origin with application in perfumery, cosmetics and household chemistry" (SYNFRA); grant No. LIDER/4/0099/L-7/15/NCBR/2016; financed by the National Centre for Research and Development in the framework of the LIDER Programme.

\section{References}

1. REGULATION (EC) No 1907/2006 OF THE EUROPEAN PARLIAMENT AND OF THE COUNCIL of 18 December 2006 concerning the Registration, Evaluation, Authorisation and Restriction of Chemicals (REACH)

2. https://www.gdrc.org/uem/lca/lca-define.html

3. K.M. Taylor, M. Weisskopf, J. Shine, Environ. Health 13(1):14 (2014)

4. D.J. Strub, J. Gibka, M. Błaszczyk, J. Kula, L. Balcerzak, M. Sikora, A. Surowiak, S. Lochyński, K. Piekarska, Synthesis, olfactory evaluation and preliminary environmental impact of citral oxime ethers (48th International Symposium on Essential Oils, Pecs, Hungary 2017)

5. https://pubchem.ncbi.nlm.nih.gov/ 
6. R. Vroman, Pediatric Toxicology: Part 3, 61 (2008)

7. S. Shahabi, S.G. Jorsaraei, A. Akbar Moghadamnia, E. Barghi, E. Zabihi, M. Golsorkhtabar Amiri, G. Maliji, A. Sohan Faraji, M. Abdi Boora, N. Ghazinejad, H. Shamsai, Cell J. 16(2), 231 (2014)

8. V. Kokkali, W. van Delft, Trends Analyt. Chem. 61, 133, (2014)

9. D. Sabaliūnas, J.R. Lazutka, I. Sabaliuniene, Environ. Pollut. 109, 251-265 (2000)

10. V.L. Jennings, M.H. Rayner-Brandes, D.J. Bird, Water Res. 35(14), 3448 (2001)

11. M. Wieczerzak, J. Namieśnik, B. Kudłak, Environ. Int. 94, 341 (2016)

12. S. Parvez, C. Venkataraman, S. Mukherji, Environ. Int. 32, 265 (2006)

13. I.D. Gaudet, Standard Procedure for MICROTOX Analysis. WCMUC (1994)

14. P. Bogaerts, J. Bohatier, F. Bonnemoy, Ecotoxicol. Environ. Saf. 49, 293 (2001)

15. P. Joly, F. Bonnemoy, J.C. Charvy, J. Bohatier, C. Mallet, Chemosphere 93(10), 2444 (2013)

16. G. Gajski, C. Ladeira, M. Gerića, V. Garaj-Vrhovaca, S. Viegas, Environ. Res. 161, 26 (2018)

17. M. Pogorzelec, K. Piekarska, Toxicity assessment of water at different stages of treatment using Microtox assay (EDP Sciences, Les Ulis, 2017)

18. H. Urbanczyk, J.C. Ast, M.J. Higgins, J. Carson, P.V. Dunlap, Int. J. Syst. Evol. Microbiol. 57, 2823 (2007)

19. K. Fent, A.A. Weston, D. Caminada, Aquat. Toxicol. 76, 122 (2006)

20. G. Gilli, T. Schiliro, Pignata, D. Traversi, E. Carraro, C. Baiocchi, R. Aigotti, D. Giacosa, E. Fea, Chemosphere 61, 1691-1699 (2005)

21. http://galfarm.com.pl/files/upload/kamfora-2.1.pdf

22. J. Rybak, Ecotoxicology: theory and laboratory practice: course in English (2011)

23. F.J. Hernández-Fernández, J.Bayo, A. Pérez de los Ríos, M.A. Vicente, F.J.Bernal, J. Quesada-Medina, Ecotoxicol. Environ. Saf. 116, 29 (2015)

24. SIDS Initial Assessment Report for 13th SIAM, Citral (2001) 